\title{
CmyView: Learning by walking and sharing social values
}

\author{
Lucila Carvalho \\ Institute of Education, Massey University, I.carvalho@massey.ac.nz \\ Cristina Garduño Freeman \\ Faculty of Architecture, Building and Planning, University of Melbourne, \\ cristina.garduno@unimelb.edu.au
}

\section{Introduction}

Networked learning practices are impacting the field of cultural heritage, both tangible and intangible, with implications for the way in which places of cultural significance are understood, managed, documented, engaged with and studied. In this chapter we explore the intersection between walking, photography, technology and learning, investigating how mobile devices can be used to foster community participation and assess social value within a networked framework for digital heritage. The chapter introduces CmyView, a mobile phone application and social media platform in development, with a design concept grounded on both digital heritage and networked learning perspectives.

In fifteen years of research and development in networked learning, we have seen a shift from its initial focus in higher education towards broader educational practices, including for example, work-based scenarios, professional development, schools and even informal learning (Hodgson, de Laat, McConnell \& Ryberg, 2014). During this time, technology has significantly evolved, transforming and extending the modalities and settings in which people learn. Ubiquitous and portable technologies nowadays enable people to connect to others and/or to learning resources anywhere, and as a result networked learning is no longer circumscribed to a specific physical space. Instead, it may take place while people are walking outdoors, traveling on public transport, eating in a café or sitting comfortably at home. Mobile computing is affecting and arguably augmenting (or at times curtailing) people's experiences of physical spaces. It not only allows for 'learning on the go' but adds to and modifies the structural composition of the physical spaces where such activities unfold, which in turn can affect the ways that people think and perceive their physical environment (Kirsh, 2013). There are new complex configurations of tools, tasks and people emerging, and these are not yet fully understood (Goodyear, Carvalho \& Dohn, 2016). The CmyView project capitalizes on mobile computing, networked practices and physical environments and spaces, to offer a way to collect, document and assess social value through embodied social practices. CmyView makes an innovative contribution to the ongoing yet critical issue, within the field of heritage, of understanding and documenting social value.

Until the 1990's heritage significance was primarily understood through expert assessment of the historic, scientific or aesthetic value of a place. Since then, community values as indicators of cultural significance have increasingly been recognised (ICOMOS, 1999). Values are important because they frame places' broader public meaning (Waterton, 2010). The field of heritage sees the concepts of social value and place as intertwined where social value is a fluid and dynamic cultural process (Smith, 2006), and place a geographical construct that incorporates people's sense of attachment to the built environment (Hayden, 1997). However, even though social value is now more readily recognised it continues to be complex to assess (Canning \& Spenneman, 2001). To date, assessment has tended to rely on social sciences methods such as surveys, workshops and interviews, forms of inquiry not usually carried out in situ (Johnston, 2003). CmyView harnesses the opportunities afforded by mobile digital technologies to design new ways to assess social value.

CmyView encourages people to collect and share their views by making images and audio recordings of personally meaningful sites they see, while walking outdoors in the natural or built environment. Each person's walking trajectory (along with their associated images and audio files) then becomes a trace-able artefact, something potentially shareable with a community. It brings together, ideas from existing mobile 'apps' that focus specifically on mapping walks (e.g. Map my Walk, Glympse or Trails) and posting/sharing photographs (e.g. Instagram, Flickr and Facebook) and extends these by adding the ability to make an audio recording that is linked to the GPS point and image taken. By facilitating the collection and sharing of information about the 
connections between people and places, CmyView also allows for a form of community curatorship of place. It engages people in observing and reflecting on their connections to built environment by prompting them to create a GPS enabled photograph, and add commentary via an audio recording. Location, image and audio are then packaged up into a shareable traceable representation of a 'walk'. Others can select a previously created 'walk', search for the places photographed by someone else by looking for these images in situ and then listen to the significance of these for the walk's creator. In so doing, CmyView offers opportunities for asynchronous situated connections between people, as walks can be made visible and catalogued in an accessible and searchable format. Coupling embodied ubiquitous practices, such as walking, with questions of social value, brings a new spatial context through which to see and make decisions around what aspects of places are valuable. Using photography as a representational embodied and creative practice to document an element of interest and distinguish it as significant, shifts the process of assessing social value from a one-way community consultation process into an informal learning network that emphasizes relationships between people and place. CmyView is both a 'tool' and 'a way' to explore places. It could be framed as digital heritage, intangible heritage, and as a form of citizen place interpretation. In thinking through the design of the system, its effect on the activity of users, its potential to contribute to the assessment of social value and its ability to develop communities around curatorial practices of place, it becomes clear that this project sits at the intersection of the fields of heritage and networked learning.

Networked learning has been described as involving learning situations in which collaboration and participation are mediated via technologies (Steeples \& Jones, 2002; Goodyear et al, 2004). Having in mind the softening of the boundaries between formal and non-formal learning, and digital and physical spaces, the notion of placebased spaces for networked learning offers a suitable framing for the CmyView project (Carvalho, Goodyear \& De Laat, 2017). The networked learning perspective highlights the opportunities for connections between people, as they assess and create (learning) resources, while experiencing both the physical and ephemeral elements and aspects of the built environment in different ways. Facets of places may become more salient when people walk around imbued with the aim of capturing sites that are of interest to them. While photography on mobile devices has become ubiquitous, the conscious task of observing, making representations and articulating the motivations for the decisions, offers an opportunity for developing visual and spatial knowledge, or even a 'good eye' (Rogoff, 2002). Observation and reflections are established methods in the pedagogical approaches of creative education (Schön, 1985). They are of particular importance for architecture students (within a formal learning context) but are also becoming pertinent for less formal learners as knowledge is increasingly dispersed across various visual, textual and spatial modalities (eg. virtual reality). Observation and reflection is an open methodology for informal learners who have an interest in issues associated with the social value of heritage sites. This opportunity is extended through intentional sharing where multiple viewpoints can engender a form of socio-visual empathy. Connections between people may arise when one person decides to experience and see the sites of interest that the walking trajectory of another may bring. CmyView creates a network of participants, each contributing to the repository of potential walks and each exploring how individuals can have significantly different forms of attachment to the same places. In this way, the assessment of social value is enabled not only by collecting data to inform government and corporate decisions, but CmyView also helps to form and inform communities' ideas about social value itself.

In this chapter, we discuss the assemblage of tasks, tools and people involved in CmyView through the ActivityCentred Analysis and Design (ACAD) framework (Goodyear \& Carvalho, 2014). The ACAD framework suggests that designing for complex learning situations involves considerations about structures of place (or elements in set design), task (or elements in epistemic design), social organization (or elements in social design), and how these, in turn, may influence activity. The framework also acknowledges that people exercise agency in reshaping or co-creating what has been designed. The chapter presents preliminary findings reporting the use of CmyView as a method for engaging with the urban environment. The next two sections present the background of our research, which includes (i) learning as social participation, bringing together the notions of situated learning, embodied cognition and networked learning, (ii) a discussion about the blurry boundaries between formal, non-formal and informal learning (iii) digital culture and heritage and issues associated with the use of mobile technologies in heritage activities of collection, preservation and interpretation of digital artefacts. Then, we introduce concepts from the ACAD framework, situating the analysis of the educational design of CmyView. This is followed by a discussion of preliminary findings of participants' interactions with (and their impressions of) the platform/methodology and the future directions of this research.

\section{Networked learning, situated learning and embodied cognition}


In line with many contemporary theories, our research acknowledges both the physically and socially situated nature of learning (e.g. Illeris, 2009). Our focus here is on learning as social participation, where people are seen as active participants in the practices of social communities, and where their identities are shaped by, and connected to, the communities in which they participate (Wenger, 2009). Drawing on the notion of situated learning we bring together activity, context and culture (Lave \& Wenger, 1991) in order to examine situations where knowledge is encountered in authentic contexts and within a community of practice. A community of practice establishes a social space for participants to discover and engage in learning partnerships related to common interests (Wenger, Trainner \& de Laat, 2011). This is particularly important for learning about the built environment where knowledge and skill is based on an iterative process of generation, reflection and observation. This social space is even more essential for learning about the social value of places, which may or may not be formally designated and recognised as sites of heritage.

In this chapter, our focus is on learning about the built environment that is mediated by mobile technologies. A networked learning perspective offers a collaborative and participatory conceptualization of learning, in which people and resources are connected via technology (Goodyear et al, 2004). However, as pointed out by Wenger, Trayner \& de Laat (2011):

Participation in a network does not require a sustained learning partnership or a commitment to a shared domain. In this sense, learning in a network does not have to have an explicit collective dimension. The learning value of network derives from access to a rich web of information sources offering multiple perspectives and dialogues, responses to queries, and help from others - whether this access is initiated by the learner or by others. (Wenger et al, 2011, p.12).

Both community and network are aspects of a social fabric, with different effects on learning in terms of value, risks, and challenges (Wenger et al, 2011). Although it is possible for community and networks to not necessarily be combined, a community will often include a network of relationships and likewise, networks will evolve and prosper because of participants' commitment to a shared interest, even if not explicitly expressed. This accords with definitions from media studies of participatory culture, which also recognizes that audiences (or networks) are not necessarily passive and can in fact operate more akin to communities in digitally mediated spaces (Jenkins, 1988; 2006). We see both community and networks as part of the social fabric in CmyView. We are particularly interested in the analysis of the connections between the design of mobile technologies for learning and the emergent activity of networked users (Goodyear, 2005). Sprake and Rogers (2014) speak of 'participatory sensing' as an emerging field of study, in which people are able to learn and teach each other about their own environments, facilitated by technology. Participatory sensing involves the ability to gather data related to personal or local enquiries, going beyond information that is provided by official sources. In this scenario, common people "can learn about and understand the world around them better and can be a part of the decision-making in improving environments for all" (p. 753). CmyView focuses on connections encouraging people to engage in social practices, on the topic area of curatorship of place. The concept design in CmyView draws our attention to the role of the physical and the 'qualities of the material' in the built environment and their effects on people's activity.

CmyView foregrounds heritage places in a non-formal learning situation, in scenarios that could be characterized as place-based spaces for networked learning (Carvalho, Goodyear \& de Laat, 2017). Place-based networked learning still involves co-creation of knowledge (Goodyear et al., 2016), and paying attention to the movement of people, objects and texts (Goodyear \& Carvalho, 2014). Co-creation of knowledge is becoming central to definitions of heritage as the importance of social value increases, and as digital media blurs the boundaries between assessment and interpretation.

The theory of embodied cognition (Clark, 2008; Kirsh, 2013) help us further theorize the potential connections between bodies, minds and technologies. Kirsh (2013) asserts that "the concepts and beliefs we have about the world are grounded in our perceptual-action experience with things, and the more we have tool mediated experiences the more our understanding of the world is situated in the way we interact through tools" (p.3:3). Cognition grounds our behavior while underpinned by our perceptual system, as we align our actions to predictions that we make about the environment (Markauskaite \& Goodyear, 2016). Embodied cognition suggests that humans think also with their bodies, not exclusively with their brains, and so interactions with tools that prescribe particular goals are likely to change the way people think and perceive. In this way, walking with the intention of recording social value through GPS, photographs and audio is distinct from walking or taking photographs for pleasure or other purposes, or simply walking for pleasure. Knowing by doing is considered more powerful than knowing by seeing (Kirsh, 2013). As we analyse the educational design of CmyView and its influence on people's activity, we examine not only design elements that may encourage 
people's social engagement as part of an emerging community, but we are also interested in people's exchanges on a topic that relates to their embodied experiences of sites. The physical trajectory, finding the site located and hearing the audio recording in situ overlays one's own experience over that of the original creator. CmyView assumes that a heterogeneous learning network is formed through participants' asynchronous interactions with others, with self-curated places (representing places of significance to them), with the physical surroundings and the 'quality of materials', which are all part of their networked interaction.

\section{Formal, non-formal and informal learning}

Formal learning is usually associated with established educational institutions, involving activities that happen as part of courses at universities, vocational training centers and schools. Such activities are often characterized as being organized and structured, and designed to meet certain learning objectives through specific experiences (OECD, 2016). Those who engage in these activities will do so with an explicit intent to gain knowledge, skills or competences. Learners' achievements will also often be measured and/or assessed (quantitatively and/or qualitatively). Informal learning, on the other hand, is not specifically organized nor structured, and it is not often associated with formal education systems. The Internet has greatly facilitated this type of learning, which is also referred to as curiosity based and self-directed (Johnson, Adams Becker, Cummins, Estrada, Freeman, \& Hall, 2016). Informal learning does not have a set of objectives, nor a clear set of learning outcomes to be achieved, measured or assessed. In between these two modes, authors sometimes also refer to a third one, characterized as non-formal learning. Non-formal learning can also be organized and structured and foregrounds learning "as a by-product of more organized activities, whether or not the activities themselves have learning objectives" (OECD, 2016, n.p.). Educational activities within a museum setting would usually fall under this category, as museum staff may devise tasks for visitor's learning, but these are not necessarily formally assessed or measured. Non-formal and informal are sometimes used interchangeably.

However, the boundaries between these descriptions are often blurry, particularly after the advent of the Internet and the emergence of mobile technologies. There has been little contention on the usefulness of such characterizations (Boys, 2010). Yet, the terms imply a neat divide between formal, non-formal and informal learning which is often "simplistically translated into spatial/representational design metaphors, rather than related through specific, situated learning and teaching practices" (Boys, 2010, p.3). The reality is more fluid and complex and importantly, the Higher Education Edition of the New Media Consortium Horizon Report states that "many experts believe that a blending of formal and informal methods of learning can create an environment that fosters experimentation, curiosity, and above all, creativity" (Johnson, Adams Becker, Cummins, Estrada, Freeman, \& Hall, 2016, p.22). The value of non-formal and informal learning in higher education is being addressed in the forthcoming policy of The European Commission who has recently issued a report describing a range of initiatives to identify ways by which informal learning activities could be evaluated and incorporated into institutions (Johnson et al, 2016). In essence, learning "may be intentional or incidental it is often both. Some learning outcomes are the intended result of participation in a study activity; some are incidental by-products of study activities; some are by-products of activities other than deliberate study" (Goodyear \& Carvalho, 2014, p. 5).

In this chapter, we discuss people's experiences with CmyView, through an example situated within a formal learning context, but as a form of non-formal or informal networked learning. The architecture students are using the app were all part of the same design studio group undertaking the same unit as part of an undergraduate course in higher education. However, the activity of using the app was outside of the prescribed curriculum and undertaken on a voluntary basis for this research. The walking activity was undertaken within the scheduled studio time and began on the university campus but asked students to explore places beyond the university campus. While the activity did not comprise of an assessment task, the exercise would arguably contribute to students' development of important abilities, related to visual skills of observation, spatial knowledge and the significance of heritage sites that they were likely already familiar with. However, the design of CmyView goes beyond the specific example in this chapter, as the app is intended to be used outside by a lay person interested in the built environment, heritage interpretation and community engagement.

The aim of CmyView is to encourage networked heritage practices and community participation, as people learn by documenting their own, and experiencing others social values of the built environment. Design principles informing the concept design for the app are closely related to networked learning key ideas, such as ways of promoting collaboration, participation and connection amongst people, and encouraging people's involvement in knowledge creation and knowledge building activities. In this case, CmyView would entail an informal (or nonformal) version of networked learning, when used as a vehicle for expanding the process of assessing social 
value. As participants contribute with their shared views via their created artefacts, they are also contributing to moving from an expert-led community consultation process - where a specialist identifies and recommends a valuable site - into an informal learning network, built by a collection of contributions by networked participants, with their multiple perspectives, dialogues, and shared understandings of the relationships between people and place.

\section{Digital culture and heritage}

Research in digital culture and heritage is mainly carried out in museum studies, usually concentrating on the digitisation of objects and places of cultural significance, the conservation of digital artefacts and the relationship between the digital and material artefacts (Kalay, Kvan, \& Affleck, 2008; Cameron \& Kenderdine 2007). Even though it has been over a decade since UNESCO (2003) promoted the international adoption of instruments for the preservation of Digital Heritage, this area of scholarship is still under researched, with little insight on the relations between social media and heritage, particularly in the topic area of participation and the contribution of information. Nevertheless, in recent years, there has been a growing movement acknowledging the significance of everyday activities as contributing to heritage (Smith, 2006).

Giaccardi (2012) points to the opportunities and transformations afforded by social media, investigating the potential links between everyday practices and forms of heritage, as manifest through online participation. Participatory culture is about the use of social media in a way that enables a "complex set of social practices that interweave memories, material traces and performative enactments to give meaning and significance in the present to the lived realities of our past" (Giaccardi, 2012, p.1). Importantly, the intersection between participatory culture and participatory media, is pointing to new ways of describing the value of, and learning about, heritage. However, understanding emerging digital heritage practices may require analysis and the reframing of online representations (Garduño Freeman, 2013; 2010). Garduño Freeman (2013; 2010) examined the significance of online representations of heritage sites, connecting numerous online representations of the Sydney Opera House (Australia) to practices of heritage and interpretation. She argues that connections between visual and material culture are evidence of the relationship people have with place and that these online instances of participatory culture do not diminish the significance of a heritage site. Instead they evidence social value. Garduño Freeman $(2013 ; 2010)$ reframes the emotional attachment that people place on buildings, such as the Sydney Opera House, and their expressions of these attachments through their use of representations, as socio-visual value arguing that posting representations and textual contributions online are new audience engagements of digital heritage. They are examples of how participatory culture manifests in a networked society, where the emergence of dispersed communities and audiences at a global level come together to participate and enact online forms of public engagement.

Three thematic areas are of critical interest for digital heritage: social practice, public formation and sense of place (Giaccardi, 2012). Social practice relates to how participatory media enables new kinds of social and visual practices, for example offering opportunities for the collection of images and production of representations, which can be used to mediate online communication, and also as expressions of personal accounts, that are, then, legitimised within communities. Public formation highlights the ways social media allows for the blurring of boundaries between community and audiences, enabling that new types of group formations emerge in the public realm. A sense of place explores experiences of social media that go beyond the online 'realm', embracing it as a way to engender and extend 'real' experiences with places of heritage.

Drawing on the notion of a sense of place in the fields of architecture and cultural geography, Giaccardi's (2012) articulation seems to touch on aspects related to research in embodied cognition (Kirsh, 2013). Social media can potentially augment the significance of traditional forms of heritage by "bring[ing] to the fore the character of place as the very matrix out of which human significance and meaning arise" (Malpas, 2008, p. 207). Conversely, social media can also augment non-traditional or everyday forms of heritage by starting with the aspects of place as touchpoints for cultural significance. Heritage scholars speak of 'community' as homogeneous collectives or groups of people with agreed viewpoints (Waterton \& Smith, 2010). 'Community' often refers to a geographically connected group of people as distinct from the term 'audiences' used to denote visitors who 'consume' but are not attached to places of heritage. In contrast to communities, 'audiences' need to be made aware of the significance of places for the local communities - they are outsiders, coming from other places, and do not have a prior connection with the site of heritage. This intellectual distinction is important because preservation is dependent on people understanding why sites of heritage are valuable (Tilden, 1977). Yet the advent of social media complicates assumptions about audiences, as people develop relationships with places via representations, both published in traditional means and those posted online, without having 
necessarily actually visited a site in person. In the CmyView project, we begin to explore participants' perspectives of a sense of place, as they visit physical sites with the intentional task of finding and registering places of personal interest. In the next section we introduce the Activity-Centred Analysis and Design (ACAD) framework, discussing specific aspects in the educational design of CmyView.

\section{Activity-centred analysis and design framework}

Inspired by ideas from architecture and design thinking, the Activity-Centred Analysis and Design (ACAD) framework (Goodyear \& Carvalho, 2014) suggests that designing for complex learning situations is best approached when connections between four main structural elements are understood; three of these elements are "designable" and one not (Figure 1). ACAD has been used to frame the analysis and design of several different types of complex learning situations including, for example, professional networks (De Laat et al, 2014); networks in higher education (Westh Nicolajsen \& Ryberg, 2014); in schools (Yeoman, 2017; Thibaut et al, 2015); libraries (Bitter-Rijpkema et al, 2014) and museums (Carvalho, 2016), and design studio spaces involving multi-user and multi-surfaces (Martinez-Maldonado et al, 2017; Thompson et al, 2013).

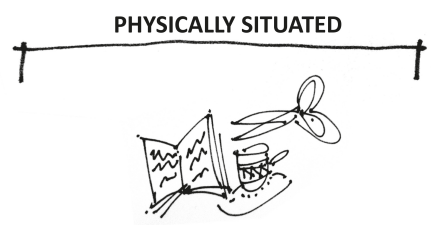

SET DESIGN
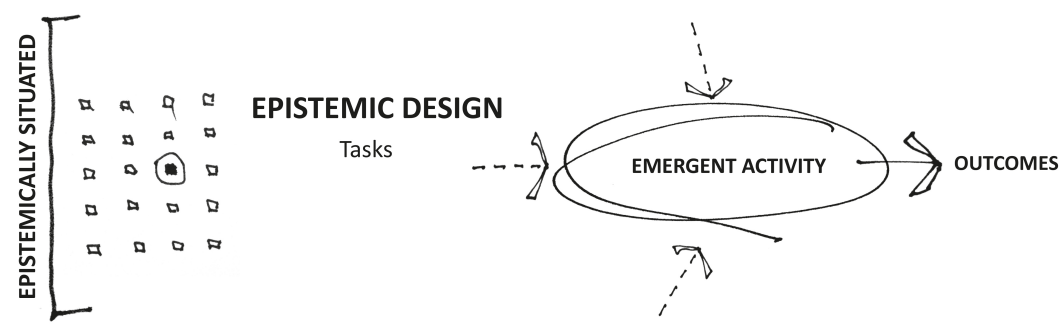

SOCIAL DESIGN

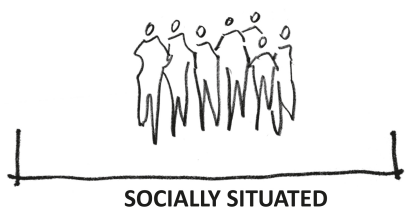

Figure 1: Activity-Centred Analysis and Design (ACAD) framework (Adapted from Goodyear \& Carvalho, 2014 p. 59)

Activity is what matters the most, it is about what people think, feel and do - an emergent process in which people exercise agency. While the framework acknowledges that design elements are likely to influence people's activity, activity cannot be entirely predicted. The four structural elements are:

(i) set design - the material and/or digital elements that are brought together to compose a learning situation - the tools, resources, artefacts and affordances of place;

(ii) epistemic design - the 'plan' for what people will do including the proposed learning tasks, along with their structuring, sequencing and pacing of how information is to be communicated to learners;

(iii) social design - social arrangements and roles, divisions of labour and who is expected to do what;

(iv) co-creation and co-configuration activity - relates the above designable components to people's activity, acknowledging that they may re-arrange and reconfigure the designed learning situation. 
The framework has been applied in the analysis of over 20 case studies, involving participation and collaborations mediated via technology in a range of complex learning situations. These include learning networks in graphic design, chemistry, teacher education and other disciplinary areas, and within different educational contexts, such as courses in higher education, schools, continuing professional development and informal learning spaces, involving online as well as place-based scenarios (Carvalho \& Goodyear, 2014, Carvalho, Goodyear, \& de Laat, 2017).

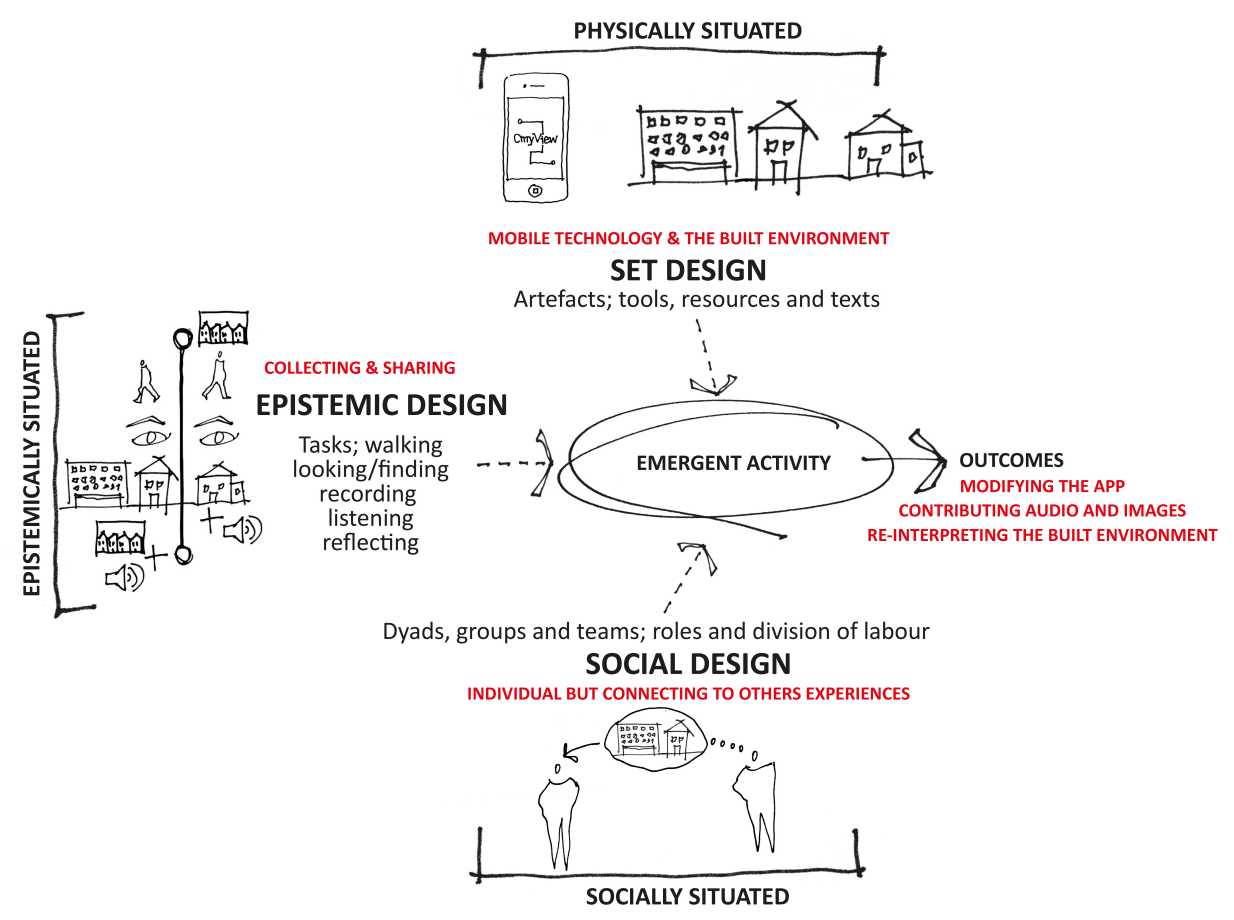

Figure 2: CmyView in the (ACAD) framework

CmyView can be analysed through the ACAD framework (Figure 2). Here, set design involves the app, deployed in a mobile technology, which users carry around. Even though the main tool participants interact with is the app, their experience will also be mediated by elements in the physical environment where the activity unfolds, as the spatial stage in which users undertake their actions. Thus, the technology is to be used in combination with elements in the physical surroundings. Similar to the blackboard, the chairs and tables in a classroom, "built forms" were not specially "brought" to the learning situation by the educational designer, albeit they are still part of the set design. In the case of CmyView, the affordances of place are very open, and might include buildings, constructions and natural elements in the environment, encountered in the user's walk.

Epistemic design relates to the proposed tasks that suggest that participants walk, observe, make a representation, and articulate why the representation was made (via recording). These are then repeated (via prompts) in the next iteration at the next point of interest. Participants not only collect but also share their views, and it is the purpose of the task and its intentionality that make this a learning task, even if in this case, participants are not being formally assessed. Epistemic design involves two main proposed tasks: (i) collecting social value and (ii) sharing social value (see Figure 3). The two tasks are structured through walking through the physical environment. In 'collecting' the task is structured through suggestions to look and record and in 'sharing' to find and listen at each 'view' in the created or followed trajectory. Both are followed by an implied opportunity for reflection on the social value of places, as the tasks invite participants to explore relationships between the built environment, personal values, and ways by which their values about the built environment can be represented and communicated in mediation with CmyView.

In the 'collecting' mode, participants are asked to take a walk in their local environment and to make 'views' during this activity. A 'view' is created when a participant takes a photograph of something significant to him/her. The possible number of 'views' is dependent on the distance walked, in other words 'views' are not immediately available, but rather, as the participant walks the opportunity for making 'views' increases, a design that purposefully encourages movement through the physical environment. The task also requests that 
participants reflect on their choices and audio record descriptions of why they chose to take that photograph. There are two main purposes for the use of audio to capture the specific meaning of the photograph. In contrast to text, audio can be recorded while the person continues to walk, thereby making the experience more fluid and less interrupted. Audio also enables emotion to be communicated and is a more intimate form of communication than text.

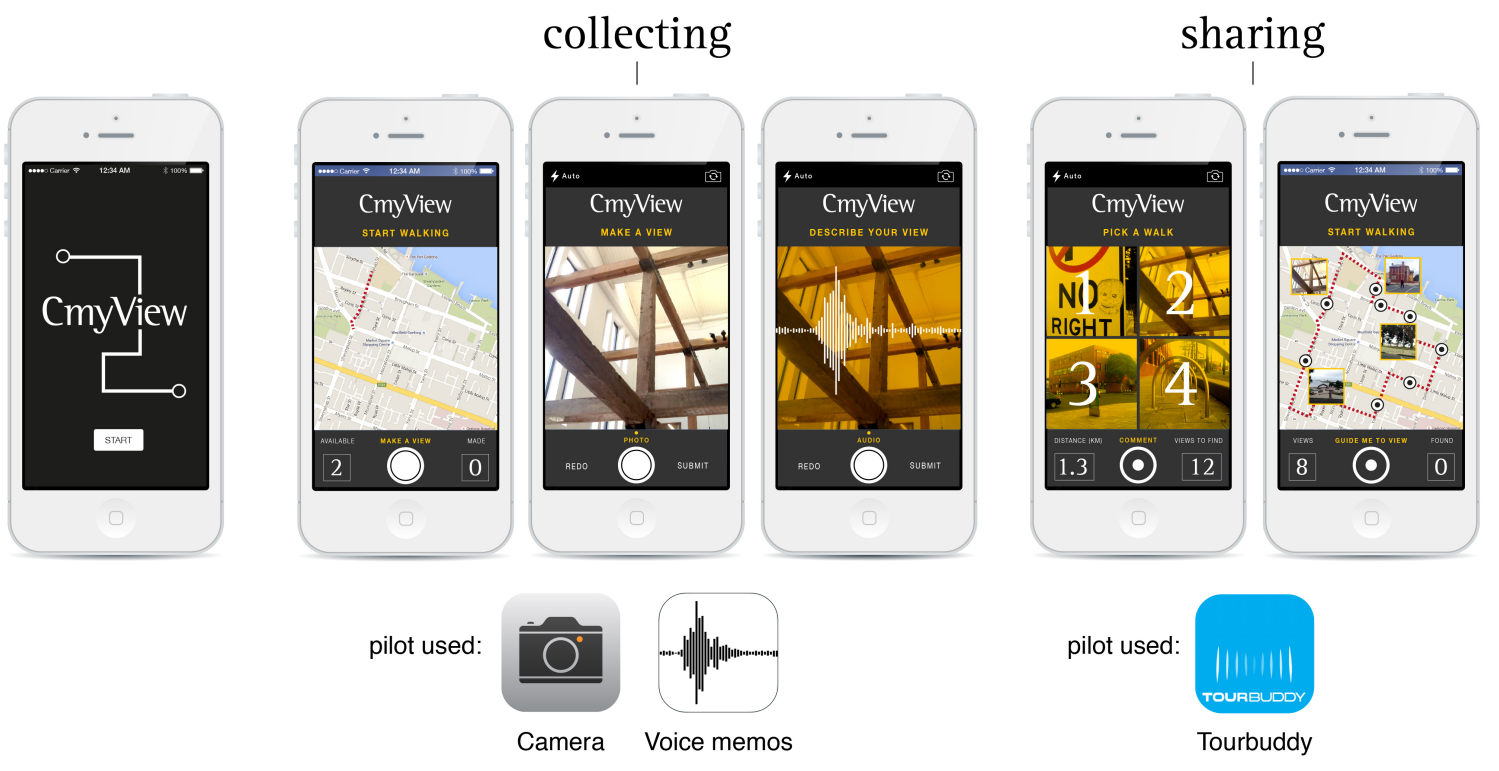

Figure 3: Collecting and Sharing Social Value

A group of 'views' made by a participant is packaged as a 'walk' that can be shared with others. In the 'sharing' mode, CmyView operates as a repository of image and audio representations of what people have found significant. In contrast to the 'collecting' mode, the epistemic design here enables an urban treasure hunt to take place. Once a 'walk' is selected, participants can follow that person's footsteps and use the GPS, visual and aural information to find or situate the photographic representation back in the physical environment. This enables digital representations to be resituated through embodied walking experiences, as the person is able to listen and learn about others memories and associations to places. Another feature in the epistemic design of CmyView relates to the collection of information for re-use. The data, both that which is gathered in the collecting mode, as well as any feedback received through the sharing mode, becomes a powerful tool to understand people's engagement with the urban environment. CmyView collects a rich layered dataset comprising three types: locational, visual and verbal that quantify intangible aspects about the built environment.

In the social design, participants are invited to asynchronously collaborate with others, while they complete their walks individually. However, other possibilities of group organisation may, nevertheless, be possible with CmyView. Diads or larger groups could work together to both identify places that are significant and together record their combined views on selected places. This aspect, would be one of the ways in which participants could co-create the experience within the overall design of CmyView.

Understanding elements in set, epistemic and social design as separate entities can at times be difficult, as they all come together as part of an assemblage. As relational elements, one enables and influences the other. The app deployed via a mobile technology (elements of set design) will tell the user what task she is to do (epistemic design), in this case collecting or sharing a view (epistemic design). As the user walks the trajectory of an invisible other (social design), again the app (set design) will alert the user to pay attention (epistemic design) to specific elements of the built environment (set design). As the user takes photographs and add their own views (co-creation and co-configuration activity), they modify the app by populating it with new elements (set design).

Overall, CmyView aims at reframing social value as dynamic, fluid and located within communities, rather than fixed in place, and shifts the role of documentation from a professional expert to participants, building upon existing social and cultural participatory practices, such as photography and walking. The focus on walking is intentional with a view of capturing its positive effect on creative thinking (Oppezzo \& Schwartz, 2014). 


\section{CmyView Prototype}

In its current stage of development CmyView was prototyped in two ways. The tasks associated with collecting mode were deployed via an iPhone app. The sharing mode was modelled through another self-curated walking app, TourBuddy, which is available by subscription. The transfer of data from the collecting to the sharing mode was carried out manually by one of the researchers. The use of this approach allowed us to rapidly capture and model experiences of participants with CmyView, and in doing so, we were able to understand and refine aspects of set, social and epistemic design before further technological development was carried out.

In the prototype version, set design involved an iPhone, with its interactive screen interface inviting people to select which mode they wish to use either collecting or sharing 'views' (social design) (see Figure 3 ). The iPhone was used in combination with elements in the built environment, as participants walked around the vicinity of the university campus.

The collecting mode begins with a standard map interface, which shows current location and maps the route walked as 'views' are made. There are 'touch-able' images that enable participants to 'make a view' and indicators on available 'views' (dependent on distance walked) and those already made. The iPhone's camera interface is used to produce a square format photograph. Once a photograph is submitted, the next screen prompts the participant to make an audio recording, about the place depicted in the photograph selected. Both the audio and photographs can be deleted and replaced whilst making the 'view'. Once made, the 'view' is then plotted on the map in the original screen.

The app used for deploying the sharing mode, currently adopts the interface of the TourBuddy app, on an iPhone and iPad. The interface offers categorisation of 'views' into 'walks'. Once a 'walk' is selected, the first view is loaded onto the screen. Directions to the location can be sought via the native Apple maps app, which loads the GPS information. The interface shows the photograph taken, the location on the map and the audio recording in one screen. TourBuddy can be enabled to automatically play the audio within a 1-5 metre radius of the GPS location.

\section{Data collection and analysis: extracts from CmyView and survey}

The methodology and the prototype version of CmyView described above were tested by four university students, enrolled in an undergraduate architecture course, who volunteered to participate in a non-formal activity that was not part of their course work. Data collection involved a collecting mode and a sharing mode. In the collecting mode, participants were instructed to take a walk and make 'views' of things in the built environment, which were of significance to them, so that together they would be documenting social value. They were intentionally not directed to specific locations, or asked to identify places of positive or negative personal value. The 'walks' were about 30 minutes long each and comprised between 6-12 'views'. We collected their photographs, audio recordings, and the geo location identifiers of each participant's walk, and afterwards, participants completed a short survey. In the sharing mode, participants were given an iPad with their four 'walks' loaded and were asked to select one of the 'walks' made by their peers. Each participant then went into the field, found the photographs of their peers whilst listening to their audio recordings and completed a second survey afterwards. The two data collection sessions were carried out in Geelong (Australia), in the immediate university surroundings, during the late afternoon one-week apart. The two online surveys (5 minutes each) used open-ended questions to elicit participants' opinions on the methodology and prototype version of CmyView.

In the ACAD framing, the students' production of photographs and audio files is part of their co-creation and co-configuration activity. They modify CmyView by populating it with visual and audio artefacts as they interact with the environment through the app (set design), completing the proposed tasks - collecting and sharing views (epistemic design), and are invited to asynchronously collaborate (social design). The audio files were transcribed and analysed together with the photographs and actual locations which were familiar to one of the researchers. The anonymous survey responses then offered insights into the experiences and reflections of the participants.

Our analysis of the audio files and photographs reveals how people ascribe different forms of attachment to places. For example, the first passage below describes historical connections between old and new aspects of a building (first passage), while the second passage highlights fluidity and contrast as facets of interest. 
I chose this second view because I feel though it incorporates sort of the new and old of what Geelong was, I suppose in the wool store days, and what has become now, in front of sort of Victoria's biggest educational institutions here, and sort of incorporating the old and new style architecture. (Walk 3 View 2) - Figure 4A

This view I was attracted to I think largely because of the straight lines that the trees have been planted in, which sort of seems to me contradicts the very sort of fluid and also maybe sharped angled nature of the branches and the leaves that are partially alive but mainly dead and crumbling. (Walk 2 View 2) - Figure 4B

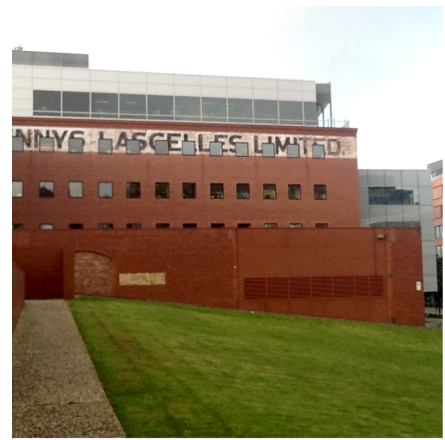

A

Walk 3 - View 2

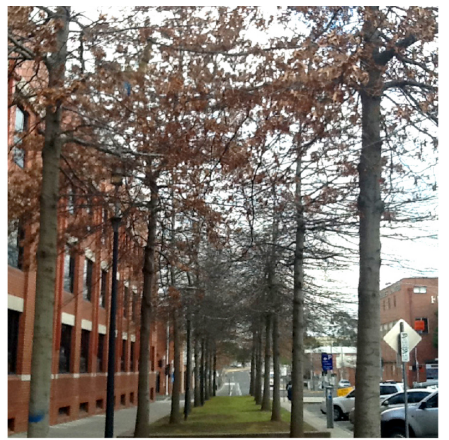

$\mathrm{B}$

Walk 2 - View 2

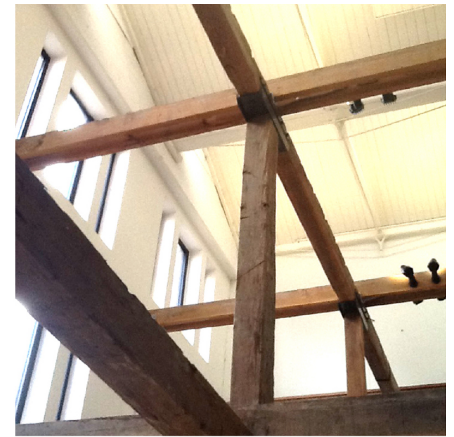

C

Walk 2 - View 1

Figure 4: Images Collected on Walks

Three themes emerged in relation to participants' experiences with CmyView: (i) changing perceptions and thinking, (ii) connections to others and (iii) extending understandings of design and architecture. In the passage below the participant acknowledges how the exercise enabled a new perspective about a familiar space.

I found interesting (...) in that it is a space that I would walk through probably everyday but it is not until you have an exercise like this where, (...) you have to stop and think about... that I really appreciate the old and new, I suppose, and you see through the old bends and then the... this clean sort of light plaster board and then also in the right hand corner the light which back lit up the bean, I thought it was really interesting. (Walk 2 View 1) - Figure 4C

Survey responses gathered several similar impressions by all four participants, where engagement in the collecting mode's proposed learning task (epistemic design) mediated by the tool (set design) seems to encourage noticing subtleties in the environment, and thinking "more critically":

I think the idea of having to explain why something stood out to you is a good one because it makes you actually stop and realise all of the beautiful things around you (Participant 1- Survey 1)

It (...) allowed me to look at certain views at a different angle, and analyse why I was attracted to these particular angles rather than others. (Participants 3 - Survey 1)

The action of taking the photograph made me consider the idea of a view more critically. Whilst when you observe with your eyes you take in the area around the view, the photo makes you be more concise with what you would like to show. Recording a memo reinforces this point of why the photo came about. (Participant 2 - Survey 1)

The second theme reflected participants' interest in connecting to others, where they welcomed the opportunity of engaging in the learning task in the sharing mode, where they indicated their experiences led to them learning about the built environment and what is valuable to others:

I am more interested in viewing other people's walks and the idea of sharing the experience and explore areas I haven't seen or thought about. (Participant 4 - Survey 1) 
Quite often we get tied up in our own constrained view of the world and by opening it up to that of [other] people is something valuable to be able to learn and understand from. (Participant 1 Survey 2)

Seeing someone else's views (or journey) was almost an intimate experience. These journeys are personal and walking on someone else's journey is just a glimpse [of] their personal take on things. It allowed me to enter into someone else's headspace and see things the way they do. (Participant 2 - Survey 2)

The third theme, focused on the participant's ability to connect their experiences of the environment gained through CmyView to other learning areas, with a particular focus on their understandings of design and architecture:

It challenged my thought process as to what was and was not worth documenting. Something that was interesting with the other person's walk that I completed was that a lot of what was captured was not specifically buildings. It broadened my understanding of built environment towards how we shape our natural environment also. (Participant 3 - Survey 2)

The way that different people see different views, helped me understand that the experiences you hope to make of your architecture may not always be successful in that respect. Your architecture will provoke a variety of experiences as different people, will experience different things driven by their different personalities. (Participant 2 - Survey 2)

Participants also reported that the overall experience elicited conversations outside the app - which is in line with the thought that in already established communities, the act of sharing experiences becomes a talking point for other types of discussions about the built environment.

\section{Conclusion and future directions}

This chapter brings together concepts that often sit at the intersection of separate fields, namely digital heritage and networked learning, to find their synergies. The chapter argues that CmyView supports the reframing of social value as dynamic, fluid and located within communities, rather than fixed in a place, reflecting networked practices and contributing to more egalitarian heritage practices. People's interaction with the app allowed them to connect to others and have an impact on how they experience their physical surroundings, through the sharing of their social values about sites of interest. The chapter argues that these activities reflect networked learning practices that cross boundaries of time and space, formal and informal learning, with people walking together while being apart, and learning by sharing.

Drawing on a framework for the analysis and design of productive learning networks, we analysed the educational design of CmyView arguing that the platform offers a space for democratic heritage education and interpretation, where participatory urban curatorship practices are nurtured, and in alignment with networked learning practices. The educational design of CmyView aims at enabling people to collaborate and participate in an ongoing dynamic activity of curating places. The ACAD framing helped us analyse ways of designing for community participation and assessment of social value. It allowed us to account for and consider the levels of complexity that are inherent in such augmented experiences. We were able to bring together, loosely coupled components in set, social and epistemic design, to analyse how they constrained and enabled the activities of participants. Part of set design (i.e. the app) could be altered and controlled, and others could not; the built environment is already in existence yet needs to be taken into account as part of the complex learning situation. Social design in this case prompted indirect collaboration between students and made us think about future possible social arrangements, potential hierarchical structure between participants, their connections, and who was viewing whose 'walks'. In epistemic design, the proposed tasks in both modes were about observation. In the collecting mode it involved careful observation of one's own experience of the built environment. In the sharing mode the task is also about observation, but in contrast it involves understanding someone else's experiences. Locating the original places photographed in an embodied task enables a kind of embodied cognition - putting the image back in context or recontextualising these places. CmyView enters a new phase of development as we fine tune the app, and explore new configurations for part of its designable elements.

\section{References}


Bitter-Rijpkema, M., Verjans, S., Didderen, W. \& Sloep, P. (2014). Biebkracht: Library professionals empowered though an inter-organisational learning network - design principles and evolution. In L. Carvalho, \& P. Goodyear (Eds.) The architecture of productive learning networks (pp. 152-167). New York: Routledge.

Cameron, F. \& Kenderdine, S. (2007). Theorizing digital cultural heritage. Cambridge: The MIT Press.

Canning, S. \& Spenneman, D. (2001). Contested space: Social value and the assessment of cultural significance in New South Wales, Australia. In M. Cotter, B. Boyd \& J. Gardiner (Eds.) Heritage Landscapes; Understanding Place and Communities (pp. 457-68). Lismore: Southern Cross University Press.

Carvalho, L. (2017). The O in MONA: Reshaping museum spaces. In L. Carvalho, P. Goodyear \& M. de Laat (Eds.) Place-based spaces for networked learning (pp. 144-159). New York: Routledge.

Carvalho, L., \& Goodyear, P. (Eds.) (2014). The architecture of productive learning networks. New York: Routledge.

Carvalho, L., Goodyear, P. \& de Laat, M. (Eds.) (2017). Place-based spaces for networked learning. New York: Routledge.

Clark, A. (2008). Supersizing the mind: Embodiment, action, and cognitive extension. Oxford: Oxford University Press.

De Laat, M., Schreurs, B., \& Sie, R. (2014). Utilizing informal teacher professional development networks using the Network Awareness Tool. In L. Carvalho, \& P. Goodyear (Eds.) The architecture of productive learning networks (pp. 239-256). New York: Routledge.

Garduño Freeman, C. (2013). Participatory culture as a site for the reception of architecture: Making a giant Sydney Opera House cake. Architecture Theory Review. 18 (3), 325-39.

Garduño Freeman, C. (2010). Photosharing on Flickr: Intangible Heritage and Emergent Publics. International Journal of Heritage Studies, 16 (4), 352-68.

Giaccardi, E. (Ed.) (2012). Heritage and social media. New York: Routledge.

Goodyear, P. (2005). Educational design and networked learning: patterns, pattern languages and design practice. Australasian Journal of Educational Technology (Online), 21(1), 82-101.

Goodyear, P. \& Carvalho, L. (2014).Framing the analysis of learning network architectures. In L. Carvalho \& P. Goodyear (Eds.) The architecture of productive learning networks (pp. 48-70). New York: Routledge.

Goodyear, P., Carvalho, L., \& Dohn, N. (2016). Artefacts and activities in the analysis of learning networks. In S. Bayne, M. de Laat, T. Ryberg, \& C. Sinclair (Eds.), Research, Boundaries and Policy in Networked Learning. New York: Springer.

Goodyear, P., Banks, S., Hodgson, V. \& McConell, D. (Eds.) (2004). Advances in research in networked learning. Dordrecht: Kluwer Academic Publishers.

Hayden, D. (1997). The power of place: Urban landscapes as public history. London: The MIT Press.

Hodgson, V., de Laat, M., McConnell, D., Ryberg, T. (Eds.) (2014). The design, experience and practice of networked learning. New York: Springer.

ICOMOS Australia. (1999). The Burra Charter: The Australia ICOMOS Charter for Places of Cultural Significance 1999.

Illeris, K. (Ed.). (2009). Contemporary theories of learning: Learning theorists - in their own words. New York: Routledge.

Jenkins, H. (2006). Convergence culture: where old and new media collide. New York: New York University Press.

Jenkins, H. (1988). 'Star Trek reread, rerun, rewritten: Fan writing as textual poaching' in Critical Studies in Mass Communications, 5(2), 85-107.

Johnston, C., Riches, L., McGregor, A. \& Buckley, K. (2003). Inspirational landscapes. Canberra: Australian Heritage Commission.

Johnson, L., Adams Becker, S., Cummins, M., Estrada, V.,Freeman, A., \& Hall, C. (2016). NMC Horizon Report: 2016 Higher Education Edition. Austin, Texas: The New Media Consortium.

Kalay, Y., Kvan, T. \& Affleck, J. (2008). New heritage: New media and cultural heritage. New York: Routledge.

Kirsh, D. (2013). Embodied cognition and the magical future of interaction design. ACM Transactions on Computer-Human Interaction, 20(1), 3:1-3:20.

Lave, J. \& Wenger, E. (1991). Situated learning: Legitimate peripheral participation. Cambridge: Cambridge University Press.

Markauskaite, L., \& Goodyear, P. (2016). Epistemic fluency and professional education: Innovation, knowledgeable action and actionable knowledge. Netherlands: Springer.

Martinez-Maldonado, R., Goodyear, P., Carvalho, L., Thompson, K., Hernandez-Leo, D., Dimitriadis, Y., Prieto, L.P., \& Wardak, D. (2017). Supporting collaborative design activity in a multi-user digital design ecology. Computers in Human Behavior, 71, 327-342. 
Rogoff, I. (2002). 'Studying Visual Culture', in N. Mirzoeff (ed.), The visual culture reader, 2nd edn, Routledge, London, pp. 24-36

Malpas, J. (2008). New media, cultural heritage and the sense of place: Mapping the conceptual ground. International Journal of Heritage Studies, 14 (3), 207.

OECD (2016) Recognition of non-formal and informal learning (Online). Available at: http://www.oecd.org/edu/skills-beyond-school/recognitionofnon-formalandinformallearning-home.htm [accessed 24 November 2016].

Oppezzo, M., \& Schwartz, D. (2014). Give your ideas some legs: The positive effect of walking on creative thinking. Journal of Experimental Psychology: Learning, Memory, and Cognition, 40 (4), 1142-52.

Schön, D. (1985) The design studio: an exploration of its traditions and potentials. London: RIBA Publications for RIBA Building Industry Trust.

Sharples, M., Taylor, J., \& Vavoula, G. (2007) A theory of learning for the mobile age. In R. Andrews and C. Haythornthwaite (Eds.), The Sage Handbook of Elearning Research. (pp. 221- 47) London: Sage.

Sprake, J. \& Rogers, P. (2014). Crowds, citizens and sensors: Process and practice for mobilising learning. Personal Ubiquitous Computing, 18(1) 753-764.

Steeples, C., and Jones, C. (eds.) (2002). Networked Learning; Perspectives and Issues. London: Springer Verlag.

Thibaut, P., Curwood, J. S., Carvalho, L. \& Simpson, A. (2015). Moving across physical and online spaces: A case study in a blended primary classroom. Learning, Media \& Technology, 40(4), 458-479.

Thompson, K., Ashe, D., Carvalho, L., Goodyear, P., Kelly, N., Parisio, M. (2013). Processing and visualizing data in complex learning environments. American Behavioral Scientist, 57(10), 1401-1420.

Tilden, F. (1977). Interpreting our heritage. Chapel Hill: University of North Carolina Press.

UNESCO. (2003). Charter on the Preservation of the Digital Heritage.

Waterton, E. (2010). The Advent of Digital Technologies and the Idea of Community.' Museum Management and Curatorship, 25 (1), pp. 5-11.

Waterton, E. \& Smith, L. (2010). The recognition and misrecognition of community heritage. International Journal of Heritage Studies, 16 (1-2), 4-15.

Wenger. E. (2009). A Social Theory of Learning. In K. Illeris (Ed.) Contemporary theories of learning: Learning theorists - in their own words. (pp. 209-217). New York: Routledge.

Wenger, E., Trayner, B., \& de Laat, M. (2011). Promoting and assessing value creation in communities and networks: a conceptual framework (Vol. 18). Heerlen: Open Universiteit.

Westh Nicolajsen, H., \& Ryberg, T. (2014). Creating a peer-driven learning network in higher education: Using web 2.0 tools to facilitate online dialogue and collaboration. In L. Carvalho, \& P. Goodyear (Eds.) The architecture of productive learning networks (pp. 94-108). New York: Routledge.

Yeoman, P. (2017). A study of correspondence, dissonance and improvisation in the design and use of a schoolbased networked learning environment. In L. Carvalho, P. Goodyear \& M. de Laat (Eds.), Place-based spaces for networked learning (pp. 41-58). New York: Routledge. 